REVISTA EDUCACIÓN LAS AMÉRICAS

FaCULTAD de EDUCACIÓN UNIVERSIDAD DE LAS AMÉRICAS
Revista Educación las Américas ISSN: 0719-7128

ccalisto@udla.cl

Universidad de Las Américas

Chile

\title{
Efecto de un calentamiento específico basado en HIIT sobre los niveles de potencia muscular en estudiantes de cuarto medio
}

Rozas Astudillo, Valeria; Pizarro San Martín, Diego; Paniagua Serey, Daniela; Huerta Ojeda, Álvaro Efecto de un calentamiento específico basado en HIIT sobre los niveles de potencia muscular en estudiantes de cuarto medio

Revista Educación las Américas, vol. 8, 2019

Universidad de Las Américas, Chile

DOI: https://doi.org/10.35811/rea.v8i0.6

Esta obra está bajo una Licencia Creative Commons Atribución-SinDerivar 4.0 Internacional. 
Artículos

\section{Efecto de un calentamiento específico basado en HIIT sobre los niveles de potencia muscular en estudiantes de cuarto medio}

Effect of a specific warm-up based on HIIT on muscle power levels in high school students

Valeria Rozas Astudillo

Universidad de Las Américas, Chile

DOI: https://doi.org/10.35811/rea.v8i0.6

Diego Pizarro San Martín

Universidad de Las Américas, Chile

Daniela Paniagua Serey

Universidad de Las Américas, Chile

Álvaro Huerta Ojeda

Universidad de Las Américas, Chile

ahuerta@udla.cl

(DD http://orcid.org/0000-0001-6871-098X

Recepción: 15 Agosto 2019

Aprobación: 23 Septiembre 2019

\section{Resumen:}

La baja aptitud física reportada por el Estudio Nacional de Educación Física sugiere que se deben desarrollar métodos innovadores y eficientes que permitan el mejoramiento de la condición física de los estudiantes. El objetivo es determinar el efecto de un entrenamiento de Intervalo de Alta Intensidad (HIIT) sobre la potencia muscular de las extremidades inferiores en estudiantes de cuarto año medio. Respecto al material y a los métodos, 28 alumnos de cuarto año medio fueron parte del estudio (Grupo Control $[\mathrm{GC}]=14 \mathrm{y}$ Grupo Experimental $[\mathrm{GE}]=14)$. Se trata de un estudio cuasi-experimental con pre y post prueba: las variables fueron la potencia muscular de las extremidades inferiores y un programa HIIT individualizado. Para el análisis estadístico se utilizó un ANOVA mixto, y como análisis post hoc una t de Student. Al término de la intervención, el programa HIIT no evidenció cambios significativos en la potencia muscular $(p>0,05)$, mientras que la prueba post hoc mostró descensos significativos en esta capacidad física en el GC ( $\mathrm{p}=0,04$; Tamaño del Efecto $=0,43)$. En conclusión, los resultados obtenidos indican que el tiempo de intervención y la carga aplicada no fueron suficientes para generar cambios en la potencia de las extremidades inferiores en estudiantes del sistema educacional chileno.

PALABRAS CLAVE: entrenamiento intervalado de alta intensidad, condición física, sistema educacional chileno, Educación Física.

\section{Abstract:}

The low physical fitness, reported by the Chilean National Study on Physical Education, suggests that innovative and efficient methods should be developed to improve the physical condition of the students. The objective of this study was to determine the effect of a High Intensity Interval Training (HIIT) on the muscular power of lower limbs in high school students. The study consisted of 28 high school students (Control Group [CG] = 14 and Experimental Group [EG] = 14). It was a Quasi-experimental study with pre and posttest. The variables were muscular power of lower limbs and an individualized HIIT. For data analysis, there were used a mixed ANOVA for statistics and Student's test as a post hoc evaluation. At the end of the intervention, the HIIT did not show significant changes in muscular power of lower limbs ( $p>0.05)$, while the post hoc test showed significant decreases in this physical capacity in the CG $(\mathrm{p}=0.04$; Effect Size $=0.43)$. The obtained results indicate that the time for the intervention and the applied load were not sufficient to generate changes in the power of lower limbs in students of the Chilean educational system. KEYWORDS: high intensity interval training, physical condition, Chilean educational system, physical education.

\section{NotAS DE AUTOR}




\section{INTRODUCCIÓN}

En Chile existe un método de evaluación externa de educación llamado Sistema de Medición de la Calidad de la Educación (SIMCE) (Agencia de Calidad de la Educación, 2015), que, en conjunto con otros sistemas, tiene como propósito comprender, interpretar y, en algunos casos, mejorar el desarrollo curricular (Monarca, 2012). En el año 2010 y con la finalidad de evaluar la condición física en estudiantes de octavo básico, se incorporó al SIMCE la asignatura de Educación Física (SIMCEEF) (Agencia de Calidad de la Educación, 2015). Este SIMCEEF nació por la iniciativa de la ley del deporte $\mathrm{N}^{\circ} 19.712$, donde se señala que "El Ministerio de Educación establecerá un Sistema Nacional de Medición de la Calidad de la Educación Física y Deportiva”, además de la creación de planes y programas para la asignatura que incluyen objetivos y contenidos que fomenten la práctica de actividad física; esta iniciativa dio lugar a que durante el año 2010 se realizaran las primeras evaluaciones nacionales del SIMCEEF, más tarde, en el 2011, se realizó la segunda evaluación con algunas modificaciones en los test de resistencia, además se incorporaron las evaluaciones antropométricas, por lo que quedó compuesta finalmente por seis test físicos y dos mediciones antropométricas (Agencia de Calidad de la Educación, 2015).

La Agencia de Calidad de la Educación (2015) expuso que la condición física está determinada por seis capacidades físicas, dentro de ellas se encuentran la fuerza, la resistencia, la flexibilidad y la coordinación, asimismo, esta misma agencia indica que la potencia aeróbica y la fuerza muscular son las capacidades más determinantes dentro de la condición física. Es así como, al término de la aplicación de SIMCEEF, se observó que los estudiantes del sistema escolar presentan bajos niveles de condición física, sobre todo en los niveles de fuerza muscular (Agencia de Calidad de la Educación, 2015). Estos antecedentes son una muestra representativa de los indicadores de condición física y salud de nuestra población, ya que en Chile casi el 90\% de los adultos y el 80\% de los adolescentes entre 15 y 19 años son sedentarios, a su vez, los estudiantes del sistema educativo están en promedio tres horas frente a un televisor, mientras que la práctica de actividad física extra programática solo es de 40 minutos por semana (Loaiza \& Atalah, 2006). Estos antecedentes reflejan la distancia que tiene nuestra población con los hábitos de vida recomendados por la Organización Mundial de la Salud (Organización Mundial de la Salud, 2010).

Martínez-Vizcaíno \& Sánchez-López (2008) postulan que la condición física es un estado y que la actividad física es una conducta, y que ambas se ven influenciadas por diversos factores, entre ellos el genotipo y el fenotipo; bajo esta consideración solo es posible manipular el fenotipo, por lo tanto, instalar algunas modificaciones en los hábitos de actividad física desde la infancia y adolescencia se vuelve relevante. Por esto, es indispensable que la adquisición de hábitos saludables se produzca durante el periodo de crecimiento, ya que la formación de hábitos en esta etapa de la vida tiende a perdurar en la edad adulta (Weisstaub, 2015). De forma paralela, la evidencia científica ha demostrado que la baja condición física y el sedentarismo se asocian a enfermedades cardiovasculares (Martínez, Del Valle, Cecchini, \& Izquierdo, 2003), y que la falta o nula práctica de actividad física acelera y determina la pérdida de las capacidades funcionales del sistema locomotor y una disminución en las respuestas adaptativas ante sobrecargas (Castillo, 2007). Consecuencia de esto es la responsabilidad de todos los agentes implicados en la formación de niños y adolescentes para cumplir con las recomendaciones mínimas sobre práctica de actividad física (Organización Mundial de la Salud, 2010).

En relación al desarrollo de la condición física en las clases de Educación Física, se han implementado diversas metodologías (Carrasco, Reigal, Ulloa, Chirosa, \& Chirosa, 2015; Huerta Ojeda et al., 2017), que van desde juegos reducidos (Carrasco et al., 2015) hasta programas de entrenamientos intervalados de alta intensidad (HIIT, por su sigla en inglés High Interval Intensity Training). Se evidencia que las clases de Educación Física, en algunas unidades específicas del currículum, requieren aplicar cargas de entrenamiento individualizadas, y que las intensidades de esta clase deben ser más altas (Curilem, Almagià, \& Yuing, 2015). En este sentido, la metodología HIIT puede ser una estrategia viable y eficaz para mejorar la condición física de los adolescentes (Costigan, Eather, Plotnikoff, Taaffe, \& Lubans, 2015). Sin embargo, no se tiene la 
certeza del resultado si se aplica un programa HIIT durante el calentamiento en una clase de Educación Física sobre la fuerza muscular de las extremidades inferiores. Consecuentemente, el propósito de este estudio fue determinar el efecto de un HIIT, desarrollado en el calentamiento específico de la clase de Educación Física, sobre la potencia muscular de las extremidades inferiores en estudiantes de cuarto año medio.

\section{ANTECEDENTES: HIIT y FUERZA MUSCULAR}

Algunos investigadores definen al HIIT como ejercicios de alta intensidad y con recuperaciones de baja intensidad (Keating et al., 2014), mientras que otros, lo describen como ejercicios vigorosos realizados a alta intensidad, pero en cortos periodos de tiempo, intercalados por intervalos de recuperación de baja a moderada intensidad o de reposo absoluto (Benito, Calderón, Peinado, \& Martínez, 2013). De esta manera, el HIIT no cuenta con un protocolo establecido, pues son tan diversos como las investigaciones que existen de él. Sin embargo, el HIIT debe ser un entrenamiento controlado y diseñado, que considere las cargas, tiempos y frecuencias para su correcta ejecución, en que se manipulen variables que incluyen intensidad, duración y recuperación, además del número de repeticiones y series (Buchheit \& Laursen, 2013).

Por su parte, la fuerza muscular se define como la capacidad del músculo para producir aceleración o deformación de un cuerpo, mantenerlo móvil o inmóvil. En algunas situaciones deportivas, la fuerza se describe como la resistencia del cuerpo del propio deportista o bien otras resistencias externas, como también la máxima tensión que manifiesta un músculo durante un tiempo determinado (González-Badillo \& Gorostiaga, 2002). De forma paralela, Gonzales \& Rivas (2002) definen la fuerza muscular como la capacidad de modificar un cuerpo en reposo o movimiento, como también la capacidad de deformar un cuerpo por compresión, es decir, juntar las moléculas de un cuerpo, o por estiramiento, alejar las moléculas de un cuerpo.

Para esta investigación se utilizó la clasificación de fuerza propuesta por García-Manzo (2000). Este investigador clasificó la capacidad física de fuerza en activa y reactiva. La primera de ellas la subdividió en Fuerza-máxima, Fuerza-veloz y Fuerza-resistencia, mientras que la segunda en Fuerza elástica-refleja y Fuerza explosiva elástica-refleja (Figura 1). 


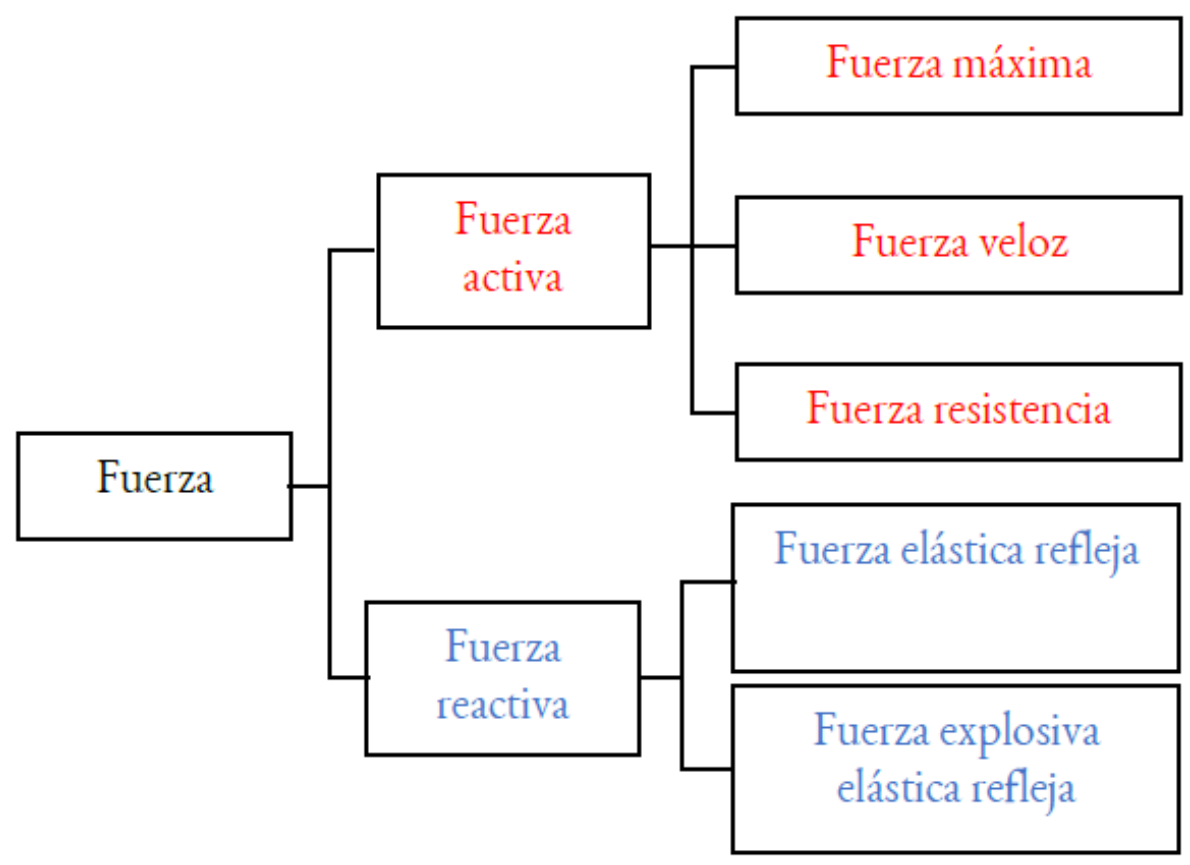

FIGURA 1

Clasificación de la capacidad de fuerza

Extraído de García-Manzo, 2000

\section{a) Fuerza Activa}

La fuerza activa involucra solamente la tensión generada de forma voluntaria por el sistema neuromuscular, y tiene dentro de sus componentes los ilustrados en la Figura 1, los que serán definidos a continuación:

Fuerza-máxima: Corresponde a la mayor tensión capaz de generar el sistema neuromuscular por medio de una acción voluntaria máxima. Esta capacidad física ha sido medida principalmente a través de una repetición máxima (1RM) (Romano et al., 2013; Tibana, Prestes, da Cunha Nascimento, \& Balsamo, 2011), 1RM indirecta (Sanchez-Medina, Perez, \& Gonzalez-Badillo, 2010) y contracciones máximas isométricas (Cadore et al., 2013).

Fuerza-veloz: Corresponde a la capacidad que posee el sistema neuromuscular de vencer una resistencia con una alta velocidad de contracción. Al igual que la fuerza máxima, esta capacidad es voluntaria y no involucra sistemas que incrementan la fuerza como el arco reflejo y los componentes en series y paralelo. Cabe mencionar que la velocidad de contracción obtenida dependerá de la carga empleada, es así como una carga muy elevada impedirá los movimientos potentes (Bautista et al., 2014; Cormie, McGuigan, \& Newton, 2010).

Fuerza-resistencia: Corresponde a la capacidad de mantener una tensión a un nivel constante por un tiempo prolongado, resistiendo el agotamiento muscular que provoca una sobrecarga de una modalidad deportiva específica. Este tipo de fuerza, al igual que la fuerza máxima y veloz, posee varias formas de medición y control (Ayala, De Ste Croix, Sainz De Baranda, \& Santonja, 2015; Ogaya, Takahashi, Shioiri, Saito, \& Okajima, 2012).

b) Fuerza Reactiva

Corresponde a una capacidad física específica, que permite desarrollar un elevado nivel de tensión muscular inmediatamente después de un intenso estiramiento mecánico de los músculos (Verkhoshansky, 1999). La fuerza reactiva involucra la tensión generada de forma voluntaria, más los componentes elásticos y la activación refleja, ambos elementos permiten incrementar los niveles de fuerza. 
El Componente elástico en serie y paralelo corresponde a un modelo presentado por Hill (1950), que busca esclarecer el proceso de la contracción muscular y la intervención de elementos no contráctiles, pero involucrados en el proceso de contracción muscular. El modelo expone al tejido muscular como un conjunto de tres elementos: un elemento contráctil, un elemento elástico situado en serie con respecto al elemento contráctil y un elemento elástico situado en paralelo con respecto a los otros dos. Las dos manifestaciones de fuerza reactiva son: a) Fuerza elástica-refleja y b) Fuerza explosiva elástica-refleja (Figura 1).

\section{MATERIAL y MÉtodos}

La muestra está compuesta por 28 alumnos de cuarto año medio (edad: 17,1 $\pm 0,5$ años; masa corporal: 66,7 $\pm 16,3 \mathrm{Kg}$ : estatura: 1,63 \pm 0,09 m), pertenecientes al Colegio Juanita Fernández de Viña del Mar, quienes fueron parte del estudio cuasi-experimental con Grupo Experimental (GE) y Grupo Control (GP). Los 28 alumnos fueron divididos en 2 subgrupos $(\mathrm{GE}=14$ y $\mathrm{GC}=14)$, que se distribuyeron por conveniencia a través de un emparejamiento muestral desarrollado luego de la aplicación del test y antes de la intervención. Los criterios de inclusión fueron: a) que todos los participantes debían pertenecer al sistema educacional chileno (cuarto medio); b) no presentar lesiones músculo-esqueléticas, patologías cardíacas preexistente o la incapacidad de ejecutar el programa de trabajo. Para la aplicación del protocolo se utilizó un diseño cuasi experimental, con pre test y post test.

\section{Procedimientos}

\section{Salto largo a pies juntos}

Para la evaluación de la potencia de las extremidades inferiores, se utilizó "Test salto largo a pies juntos" con el objetivo de evaluar la fuerza explosiva de las extremidades inferiores (Cancino, Castillo, \& Huerta Ojeda, 2013), lo que se aprecia en la Figura 2. El protocolo utilizado consideró tres intentos por participante, y se dejó el con mayor rendimiento para el emparejamiento muestral, el análisis de datos y planificación individualizada

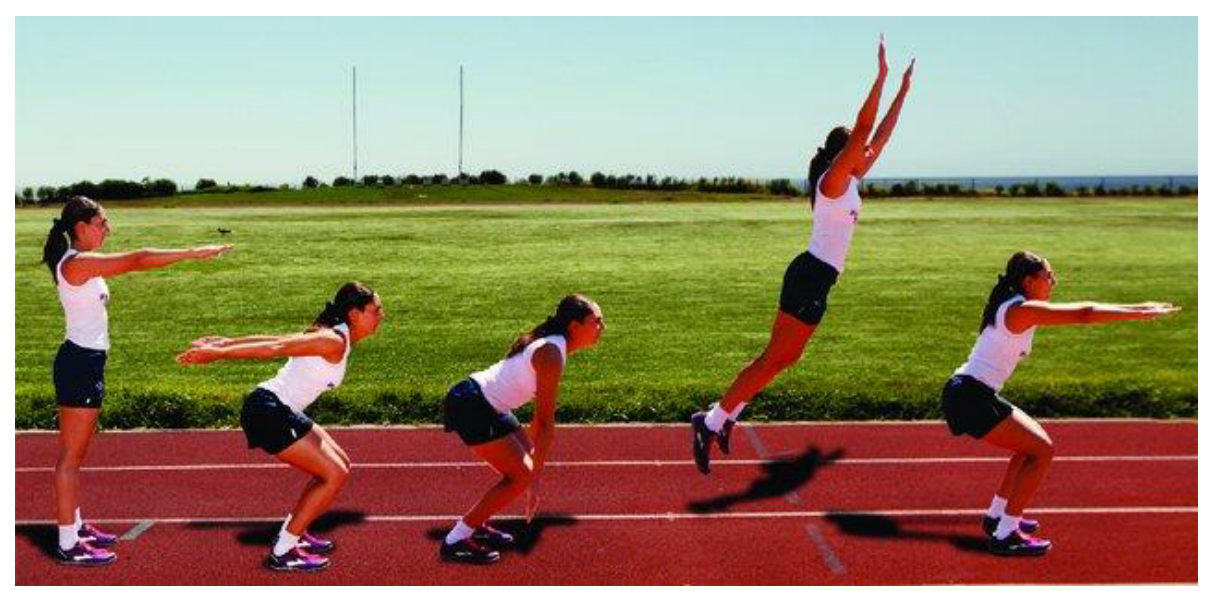

FIGURA 2

Salto largo a pies juntos

Cancino et al., 2013.

\section{Programa de intervención}

El programa de intervención consideró ocho microciclos, con una sesión semanal. En cada una de estas sesiones se trabajó la potencia muscular de las extremidades inferiores con un método de entrenamiento HIIT individualizado para cada estudiante. Todas las sesiones fueron desarrolladas de forma progresiva 
(desde el 50 hasta el 80\% de la repetición máxima (RM) o capacidad máxima de salto) (Tabla 1). Cada repetición fue diseñada de forma individualizada, y se determinó la longitud del salto para cada participante en relación al test de salto largo, considerado como el 100\% de la RM. El tiempo de trabajo total fue de 15 minutos, mientras que el tiempo efectivo de trabajo fue de 10 minutos, a su vez, el tiempo de cada serie fue de 40 segundos, mientras que la pausa entre series fue de 60 segundos y el descanso de 90 segundos. Las sesiones no consideraron un calentamiento, ya que la intervención formó parte de dicho calentamiento.

TABLA 1

Estructura del programa HIIT en base a al test de Salto largo a pies juntos

\begin{tabular}{|c|c|c|c|c|c|c|c|c|c|c|}
\hline Sesiones & 1 & 2 & 3 & 4 & 5 & 6 & 7 & 8 & 9 & 10 \\
\hline Objetivos & E & I & I & I & I & I & I & I & I & \\
\hline Intensidad & $100 \%$ & $50 \%$ & $55-60-60 \%$ & $60-65-70 \%$ & $65-70-75 \%$ & $70-75-80 \%$ & $60-70-75 \%$ & $65-70-75 \%$ & $70-75-80 \%$ & $100 \%$ \\
\hline Series & 1 & 3 & $\begin{array}{l}2 \times 50 \% 2 \\
\times 55 \% 2 \times \\
60 \%\end{array}$ & $\begin{array}{l}2 \times 60 \% 2 \\
\times 65 \% 2 \times \\
70 \%\end{array}$ & $\begin{array}{l}2 \times 65 \% 2 \\
\times 70 \% 2 \times \\
75 \%\end{array}$ & $\begin{array}{l}2 \times 70 \% 2 \\
\times 75 \% 2 \times \\
80 \%\end{array}$ & $\begin{array}{l}2 \times 60 \% 2 \\
\times 70 \% 2 \times \\
75 \%\end{array}$ & $\begin{array}{l}2 \times 65 \% 2 \\
\times 70 \% 2 \times \\
75 \%\end{array}$ & $\begin{array}{l}2 \times 70 \% 2 \\
\times 75 \% 2 \times \\
80 \%\end{array}$ & 1 \\
\hline Repeticiones & 1 & 15 & 151210 & 12108 & 10810 & 864 & 12106 & 1086 & 864 & 1 \\
\hline Pausa (S) & 60 & 60 & 60 & 60 & 60 & 60 & 60 & 60 & 60 & 60 \\
\hline $\begin{array}{l}\text { Macropausa } \\
\text { (S) }\end{array}$ & 90 & 90 & 90 & 90 & 90 & 90 & 90 & 90 & 90 & 90 \\
\hline $\begin{array}{l}\text { Volumen } \\
\text { (saltos) }\end{array}$ & 1 & 45 & 74 & 60 & 56 & 36 & 56 & 48 & 36 & 1 \\
\hline
\end{tabular}

Fuente: elaboración propia.

HIIT: entrenamiento intermitente de alta intensidad; E: evaluación; I: Intervención; s: segundos; \%: por ciento.

\section{Análisis estadistico}

Tanto los datos del GE como del GC en pre y post test fueron sometidos a la prueba de normalidad de Shapiro-wilk. Posteriormente, para observar cambios en la potencia muscular de las extremidades inferiores se aplicó un ANOVA mixto. Como análisis post Hoc se usó la prueba t de Student, mientras que el tamaño del efecto fue calculado con la prueba $\mathrm{d}$ de Cohen. Este análisis considera un efecto insignificante $(\mathrm{d}<0,2)$, pequeño $(d=0,2$ hasta 0,6$)$, moderado $(d=0,6$ a 1,2$)$, grande $(d=1,2$ a 2,0$)$ o muy grande $(d>2,0)$. El nivel de significancia para cada uno de los análisis estadísticos fue de $\mathrm{p}<0,05$. El análisis estadístico se realizó con el software Startical Product and Service Solutions (SPSS). El nivel de significancia para todos los datos fue de $\mathrm{p}<0,05$.

\section{Resultados}

Aplicada la prueba ANOVA, no se evidenciaron cambios significativos en la potencia muscular de las extremidades inferiores entre el GE y GC antes y después de la aplicación del programa HIIT $(p>0,05)$. En relación al análisis post hoc, el GE evidenció cambios significativos entre el pre test y post test $(\mathrm{p}>0,05$; TE $=0,11)$ en la distancia de salto $(\mathrm{m})$ en el Test de Salto Largo a Pies Juntos. De forma paralela, el GC tuvo un descenso significativo en la potencia muscular de las extremidades inferiores $(p<0,05$; TE $=0,43)$. Las progresiones y los cambios producidos entre el pre test y post test, se encuentran reportados en Figura $3 \mathrm{y}$ en las Tabla 2 y Tabla 3. 
TABLA 2

Potencia muscular de las extremidades inferiores antes y después de la aplicación de programa HIIT

\begin{tabular}{|c|c|c|c|c|c|}
\hline \multicolumn{6}{|l|}{ ANOVA } \\
\hline & GE & & $\mathrm{GC}$ & & \\
\hline & $\begin{array}{l}\text { pre test } \\
\text { media } \\
\pm \mathrm{DS}\end{array}$ & $\begin{array}{l}\text { post } \\
\text { test } \\
\text { media } \\
\pm \text { DS }\end{array}$ & $\begin{array}{l}\text { pre } \\
\text { test } \\
\text { media } \\
\pm \text { DS }\end{array}$ & $\begin{array}{l}\text { post } \\
\text { test } \\
\text { media } \\
\pm \mathrm{DS} \\
\end{array}$ & $\begin{array}{l}\text { ANOVA } \\
\text { mixto }\end{array}$ \\
\hline $\begin{array}{l}\text { distancia } \\
\text { (m) }\end{array}$ & $\begin{array}{l}1,64 \pm \\
0,38\end{array}$ & $\begin{array}{l}1,59 \pm \\
0,41\end{array}$ & $\begin{array}{l}1,68 \pm \\
0,36\end{array}$ & $\begin{array}{l}1,53 \pm \\
0,34\end{array}$ & $\mathrm{~ns}$ \\
\hline
\end{tabular}

Fuente: elaboración propia.

GE: Grupo Experimental; GC: Grupo Control; m: metros; DS: desviación estándar; ns: no significativo.

TABLA 3

Análisis post hoc para la distancia en test Salto Largo a Pies Juntos del GE y GC.

\begin{tabular}{|c|c|c|c|c|c|c|}
\hline \multicolumn{7}{|c|}{ Grupo Control } \\
\hline & $\begin{array}{l}\text { pre } \\
\text { test } \\
\text { media } \\
\pm \\
\text { DS }\end{array}$ & $\begin{array}{l}\text { post } \\
\text { test } \\
\text { media } \\
\pm \\
D S\end{array}$ & $\begin{array}{l}\Delta \text { pre } \\
y \\
\text { post } \\
\text { test }\end{array}$ & $\begin{array}{l}\text { Diferencia } \\
\text { porcentual }\end{array}$ & $\begin{array}{l}\text { t de } \\
\text { Student }\end{array}$ & $\begin{array}{l}\mathrm{d} \text { de } \\
\text { Cohen }\end{array}$ \\
\hline $\begin{array}{l}\text { distancia } \\
\text { (m) }\end{array}$ & $\begin{array}{l}1,68 \\
\pm \\
0,36\end{array}$ & $\begin{array}{l}1,53 \\
\pm \\
0,34\end{array}$ & $-0,15$ & $-8,92 \%$ & * & 0,43 \\
\hline \multicolumn{7}{|c|}{ Grupo Experimental } \\
\hline & $\begin{array}{l}\text { pre } \\
\text { test } \\
\text { media } \\
\pm \\
\text { DS }\end{array}$ & $\begin{array}{l}\text { post } \\
\text { test } \\
\text { media } \\
\pm \\
\text { DS }\end{array}$ & $\begin{array}{l}\Delta \text { pre } \\
y \\
\text { post } \\
\text { test }\end{array}$ & $\begin{array}{l}\text { Diferencia } \\
\text { porcentual }\end{array}$ & $\begin{array}{l}\text { t de } \\
\text { Student }\end{array}$ & $\begin{array}{l}\mathrm{d} \text { de } \\
\text { Cohen }\end{array}$ \\
\hline $\begin{array}{l}\text { distancia } \\
\text { (m) }\end{array}$ & $\begin{array}{l}1,64 \\
\pm \\
0,38\end{array}$ & $\begin{array}{l}1,59 \\
\pm \\
0,41\end{array}$ & $-0,05$ & $-3,04 \%$ & ns & 0,11 \\
\hline
\end{tabular}

Fuente: elaboración propia.

GE: Grupo Experimental; GC: Grupo Control; m: metros; DS: desviación estándar; $\Delta$ : delta; ns: no significativo; ${ }^{*} \mathrm{p}<0,05$. 
Salto largo a pies junto en pre y post test

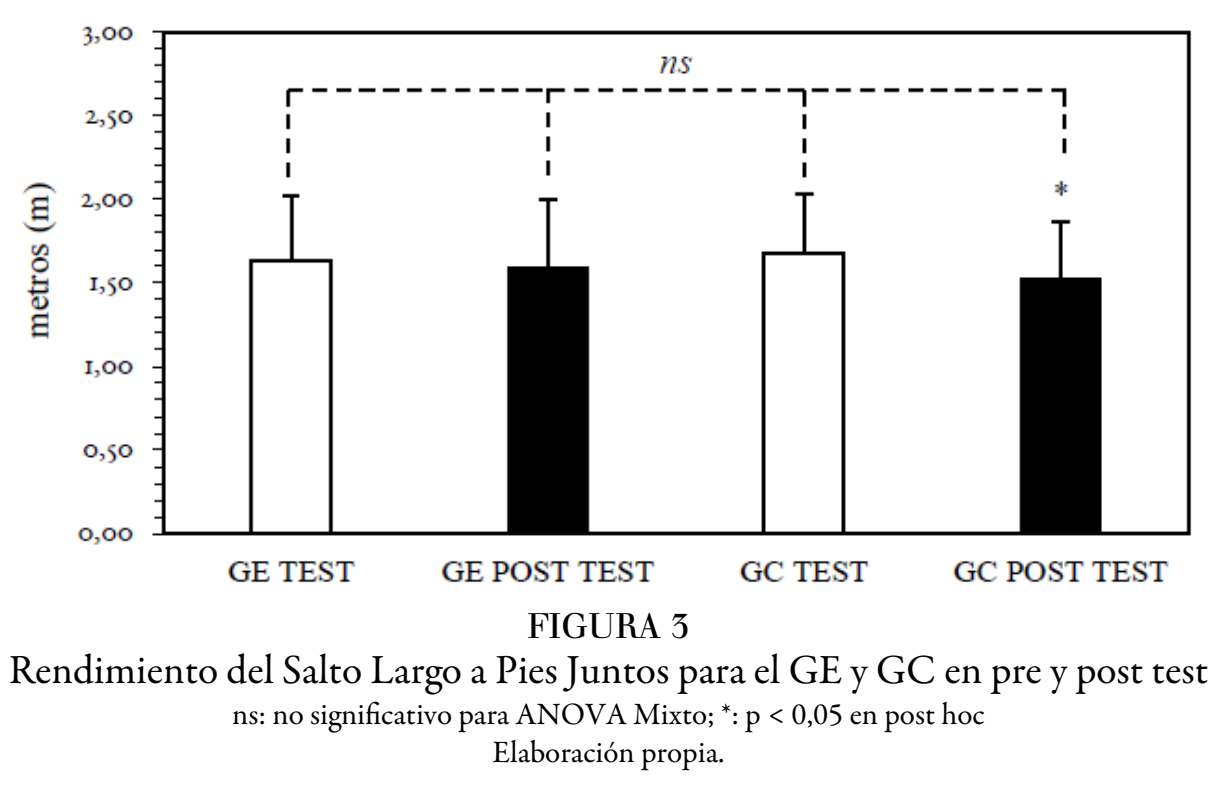

\section{DisCUSIóN}

En relación al propósito de este estudio, determinar el efecto de un HIIT desarrollado en el calentamiento específico de la clase de Educación Física sobre la potencia muscular de las extremidades inferiores en estudiantes de cuarto año medio, los resultados indicaron que el GE no mostró cambios significativos una vez aplicado el protocolo $(\mathrm{p}>0,05)$. Independiente de los resultados obtenidos en este estudio, es difícil realizar una comparación con investigaciones similares, ya que hasta donde el conocimiento alcanza, otras investigaciones que han aplicado un HIIT como variable independiente, también han declarado formas distintas de aplicarlo; esto hace alusión a que la carga de entrenamiento (intensidad, volumen, pausa y/o densidad) varía entre los distintos protocolos utilizados (Chelly et al., 2010; Michailidis et al., 2013; Ozbar, Ates, \& Agopyan, 2014). Un ejemplo de lo mencionado anteriormente fue la investigación desarrollada por Chelly et al. (2010), investigadores que evidenciaron un aumento significativos en la potencia de salto (Squat Jump - Counter Movement Jump), sin embargo, estos resultados presentan dos diferencias importantes al ser comparados con el presentes estudio; por un lado, los vectores de fuerza fueron distintos (Jiménez-Reyes et al., 2018), mientras que por otro, no utilizaron un HIIT durante el calentamiento específico. Da forma paralela, Michailidis et al. (2013) reportaron diferencias significativas en la potencia de las extremidades inferiores posterior a 12 semanas de entrenamientos pliométricos $(\mathrm{p}<0,05)$; posiblemente estas 12 semanas de entrenamiento hayan marcado la diferencias en los resultados obtenidos. En otra investigación, se reportó un incremento en la potencia muscular luego de ocho semanas de entrenamiento pliométrico $(\mathrm{p}<0,05)$; en este caso los investigadores aplicaron más de un entrenamiento semanal, por lo que la cantidad de estímulos semanales fue una de las razones para generar cambios en las capacidades físicas (Ozbar et al., 2014). Por estos antecedentes, quizás las ocho semanas, el bajo porcentaje de la RM, la baja cantidad de repeticiones y/o la pausa utilizada en el protocolo HIIT sean los factores que influyeron en los cambios no significativos de la potencia muscular en las extremidades inferiores en estudiantes de cuarto año medio.

Sumado a lo anterior, un dato importante que se debe analizar es que la gran mayoría de los estudios consultados están relacionados a deportes (Chelly, Hermassi, Aouadi, \& Shephard, 2014; Hall, Bishop, \& Gee, 2016; Mackala \& Fostiak, 2015). En cada uno de estos estudios se han reportados incrementos significativos en la potencia muscular de las extremidades inferiores. Sin embargo, en ninguna de estas 
investigaciones se utilizó un HIIT basado en saltos horizontales, como tampoco se incorporó esta metodología en el calentamiento específico de la clase de Educación Física para incrementar la potencia muscular. Por lo anterior, y dada la escasa cantidad de investigaciones que han investigado el HIIT en el sistema educacional chileno (Maliqueo, Huerta Ojeda, Ríos, Serrano, \& Amigo, 2017; Huerta Ojeda et al., 2017) es difícil discutir los hallazgos y generalizar los resultados.

Como fue mencionado en párrafos anteriores, el HIIT es una estrategia eficiente para incrementar la salud muscular de la población (Costigan et al., 2015), pues tiene como característica principal que los entrenamientos pueden ser ejecutados en un periodo corto de tiempo al ser comparado con los entrenamientos aeróbicos tradicionales (Keating et al., 2014). Independiente de aquello, como en todas las contracciones músculo-esqueléticas, durante la aplicación de un HIIT están presente las tres vías energéticas que proveen de Adenosín Trifosfato al sistema neuromuscular; sin embargo, se asume que la contribución de anaeróbica láctica y la descarga neuromuscular para generar óptimas tensiones músculo-esqueléticas son las variables secundarias más importantes a considerar en el presente estudio; desafortunadamente, no existe una técnica sólida (gold standard) para medir su contribución durante la aplicación del HIIT en terreno; en este sentido, es importante destacar que la medición del déficit de oxígeno y la concentración de lactato muscular, esta última evaluada en sangre capilar, tienden a ser los métodos de valoración más recurrentes por la mayoría de los investigadores (Buchheit \& Laursen, 2013). En relación a lo anterior y considerando los principios fisiológicos del HIIT, en el presente estudio no fue posible evidenciar en terreno la vía energética predominante, pero en la descripción de la naturaleza de las contracciones musculares y de los porcentajes de cada uno de los saltos usados en el protocolo HIIT de esta investigación, se asume que la vía anaeróbica aláctica (ATP-PC) fue la encargada de sustentar las diferentes sesiones de entrenamiento. Al término de la intervención, siempre que se evidencien cambios significativos luego de la aplicación de un HIIT, se asume una mayor aptitud cardiorrespiratoria y capacidad de trabajo, mayor biogénesis mitocondrial muscular y niveles de transportadores de glucosa (GLUT-4), incluyendo una mejor sensibilidad a la insulina (Keating et al., 2014).

Independiente de los resultados reportados en el presente estudio, es importante mencionar que al aplicar un método de alta intensidad los sujetos deben estar libre de lesiones. Un ejemplo de lo anterior, son los resultados presentados por ter Stege, Dallinga, Benjaminse, \& Lemmink (2014), estos investigadores reportaron una reducción del valgo de rodilla posterior a un entrenamiento neuromuscular a través de ejercicios pliométricos. A su vez, Xu, Lombardi, Jiao, \& Banfi (2016), en una revisión sistemática, concluyeron que los ejercicios de alta intensidad aumentan la masa ósea en mujeres jóvenes, y que estos ejercicios también podrían ser útiles en los hombres, promocionando la salud ósea y la prevención de la osteoporosis a temprana edad. En otros estudios con metodología HIIT, se ha concluido que este tipo de entrenamientos aumenta el rendimiento de velocidad ( $\mathrm{p}<0,001$ ) (Kohn, Essén-Gustavsson, \& Myburgh, 2011; Sperlich et al., 2011), fuerza explosiva en counter movement jump ( $<<0,001)$ (Brocherie, Girard, Faiss, $\&$ Millet, 2015), lactato deshidrogenasa (LDH) homogeneizado ( $p<0,05)$ y fibras tipo IIa $(\mathrm{p}<0,05)$ (Kohn et al., 2011). Pese a los buenos resultados demostrados con el método HIIT en los deportistas (Brocherie et al., 2015; Kohn et al., 2011; Sperlich et al., 2011), ninguna de estas investigaciones mencionadas incluyó un HIIT en el calentamiento específico de la clases Educación Física.

Por lo descrito en los párrafos anteriores, los beneficios del entrenamiento pliométrico y de un método HIIT han sido explícitos en el rendimiento deportivo, pero los reportes asociados de esta metodología innovadora de entrenamiento en las clases de Educación Física son escasos y quizás un aumentos en las sesiones semanales junto a un incremento en el volumen de la carga podrían mejorar la potencia de las extremidades inferiores de estudiantes de enseñanza media del sistema educacional chileno. 


\section{Conclusión}

En relación a la información recolectada antes y después de la aplicación de un programa HIIT, se pudo observar cambios no significativos en la potencia de las extremidades inferiores. Es posible que el tiempo de intervención y la carga aplicada no fueron suficientes para generar cambios en la distancia horizontal, asociada a la fuerza explosiva de las extremidades inferiores, en estudiantes del sistema educacional chileno.

Perspectivas futuras

Considerando los resultados evidenciados, para una próxima intervención se deberían tener en cuenta las siguientes consideraciones: a) una muestra más numerosa; b) aumentar las semanas de intervención; c) aumentar en número de intervenciones por semana; y d) adecuar las pausas del programa en relación al volumen de trabajo.

Si bien, esta investigación no reportó cambios significativos en la fuerza explosiva de las extremidades inferiores de estudiantes del sistema educacional chileno, es importante incentivar a profesores de Educación Física a que adopten métodos de entrenamiento innovadores y de bajo costo para el desarrollo de la fuerza muscular. De esta forma, se podrán enseñar contendidos del currículum no solo en la unidad de acondicionamiento físico en la enseñanza básica y la unidad de enteramiento para la vida en enseñanza media, sino que también en cada unidad de aprendizaje, contribuyendo a desarrollar hábitos de vida en cada uno de los estudiantes de nuestro sistema educacional.

\section{REFERENCIAS}

Agencia de Calidad de la Educación. (2015). Informe Estudio Nacional de Educación Física. $8^{\circ}$ básico. Recuperado de https://www.agenciaeducacion.cl/estudios/estudio-de-educacion-fisica/

Ayala, F., De Ste Croix, M., Sainz De Baranda, P., \& Santonja, F. (2015). Acute effects of two different stretching techniques on isokinetic strength and power. Revista Andaluza de Medicina del Deporte, 8(3), 93-102. https:// doi.org/10.1016/j.ramd.2014.06.003

Bautista, I.J., Chirosa, I.J., Chirosa, L. J., Martín, I., González, A., \& Robertson, R. J. (2014). Development and validity of a scale of perception of velocity in resistance exercise. Journal of Sports Science and Medicine, 13(3), 542-549.

Benito, P., Calderón, F., Peinado, A., \& Martínez, I. (2013). VI International Symposium in Strength Training. (F. de C. de la A. F. y del D.-U. P. de Madrid, Ed.). Madrid.

Brocherie, F., Girard, O., Faiss, R., \& Millet, G. (2015). High-intensity intermittent training in hypoxia: A doubleblinded, placebo-controlled field study in youth football players. Journal of Strenght and Conditioning Research, 29(1), 226-237.

Buchheit, M., \& Laursen, P. B. (2013). High-intensity interval training, solutions to the programming puzzle Part II: Anaerobic energy, neuromuscular load and practical applications. Sports Medicine, 43(10), 927-954. https:// doi.org/10.1007/s40279-013-0066-5

Cadore, E., Pinheiro, E., Izquierdo, M., Correa, C., Radaelli, R., Martins, J., ... Pinto, R. (2013). Neuromuscular, Hormonal, and Metabolic Responses to different plyometric training volumes in rugby players. The Journal of Strength and Conditioning Research, 27(11), 3001-3010.

Cancino, J., Castillo, N., \& Huerta Ojeda, Á. (2013). Ejercicio y Condición Física. Valparaíso: Escuela Naval Arturo Prat, Ed.

Carrasco, H., Reigal, R., Ulloa, D., Chirosa, I. J., \& Chirosa, L. J. (2015). Efecto de los juegos reducidos en la composición corporal y la condición física aeróbica en un grupo de adolescentes escolares. Revista Médica de Chile, 143(6), 744-750. https://doi.org/10.4067/S0034-98872015000600007

Castillo, M. J. (2007). La condición física es un componente importante de la salud para los adultos de hoy y del mañana. Selección, 17(1), 2-8. Recuperado de https://www.cafyd.com/selec0701/Selultimo.pdf 
Chelly, M. S., Ghenem, M. A., Abid, K., Hermassi, S., Tabka, Z., \& Shephard, R. J. (2010). Effects of in-season shortterm plyometric training program on leg power, jump- and sprint performance of soccer players. Journal of Strength and Conditioning Research, 24(10), 2670-2676. https://doi.org/10.1519/JSC.0b013e3181e2728f

Chelly, M. S., Hermassi, S., Aouadi, R., \& Shephard, R. J. (2014). Effects of 8-week in-season plyometric training on upper and lower limb performance of elite adolescent handball players. Journal of Strength and Conditioning Research, 28(5), 1401-1410. https://doi.org/10.1519/JSC.0000000000000279

Cormie, P., McGuigan, M. R., \& Newton, R. U. (2010). Adaptations in athletic performance after ballistic power versus strength training. Medicine and Science in Sports and Exercise, 42(8), 1582-1598. https:// doi.org/10.1249/MSS.0b013e3181d2013a

Costigan, S. A., Eather, N., Plotnikoff, R. C., Taaffe, D. R., \& Lubans, D. R. (2015). High-intensity interval training for improving health-related fitness in adolescents: A systematic review and meta-analysis. British Journal of Sports Medicine, 49(19), 1253-1261. https://doi.org/10.1136/bjsports-2014-094490

Curilem, C., Almagià, A., \& Yuing, T. (2015). Aplicación del test course navette en escolares. Revista Motricidad Humana, 16(2), 95-99. Recuperado de http://www.revistamotricidad.com/wp-content/ uploads/2016/03/95-99.pdf

García-Manzo, J. (2000). La fuerza: Fundamentación, valoración y entrenamiento. Madrid: Gimnos Ed.

Gonzales, J., \& Rivas, J. (2002). Rendimiento Deportivo "Bases de la programación del entrenamiento de la fuerza." Barcelona: INDE.

González-Badillo, J., \& Gorostiaga, E. (2002). Fundamentos del entrenamiento de la fuerza: Aplicación al alto rendimiento. (3ra edición). Barcelona: INDE.

Hall, E., Bishop, D. C., \& Gee, T. I. (2016). Effect of plyometric training on handspring vault performance and functional power in youth female gymnasts. PLOS ONE, 11(2), 1-10. https://doi.org/10.1371/ journal.pone. 0148790

Hill, A. (1950). The series elastic component of muscle. Proceedings of the Royal Society of London. Series B, Biological Sciences, 137(887), 273-280.

Huerta Ojeda, Á., Maliqueo, S. G., Guerra, C. M., Barahona-Fuentes, G., Villanueva, R. T., \& Serrano, P. C. (2017). Efectos de un entrenamiento intervalado de alta intensidad en la capacidad aeróbica de adolescentes. Revista Médica de Chile, 145, 972-979.

Jiménez-Reyes, P., Samozino, P., García-Ramos, A., Cuadrado-Peña, V., Brughelli, M., \& Morin, J. (2018). Relationship between vertical and horizontal force-velocity-power profiles in various sports and levels of practice. PeerJ, 6(e5937), 1-18. https://doi.org/10.7717/peerj.5937

Keating, S. E., Machan, E. A., O’Connor, H. T., Gerofi, J. A., Sainsbury, A., Caterson, I. D., \& Johnson, N. A. (2014). Continuous exercise but not high intensity interval training improves fat distribution in overweight adults. Journal of Obesity, 1, 1-12. https://doi.org/10.1155/2014/834865

Kohn, T. A., Essén-Gustavsson, B., \& Myburgh, K. H. (2011). Specific muscle adaptations in type II fibers after highintensity interval training of well-trained runners. Scandinavian Journal of Medicine and Science in Sports, 21(6), 765-772. https://doi.org/10.1111/j.1600-0838.2010.01136.x

Loaiza, S., \& Atalah, E. (2006). Factores de riesgo de obesidad en escolares de primer año básico de Punta Arenas. Revista Chilena de Pediatría, 77(1). http://dx.doi.org/10.4067/S0370-41062006000100003.

Mackala, K., \& Fostiak, M. (2015). Acute effects of plyometric intervention-performance improvement and related changes in sprinting gait variability. Journal of Strength and Conditioning Research, 29(7), 1956-1965.

Maliqueo, S. G., Huerta Ojeda, Á., Ríos, L. C., Serrano, P. C., \& Amigo, T. R. (2017). Efecto de un método de Entrenamiento Intervalado de Alta Intensidad sobre el consumo máximo de oxígeno en escolares chilenos. Revista Universidad y Salud, 19(3), 359-365. https://doi.org/10.22267/rus.171903.98

Martínez-Vizcaíno, V. \& Sánchez-López, M. (2008). Relación entre actividad física y condición física en niños y adolescentes. Revista Española de Cardiología, 61(2), 108-111. https://doi.org/10.1157/13116196

Martínez, A., Del Valle, M., Cecchini, J. A., \& Izquierdo, M. (2003). Asociación de la condición física saludable y los indicadores del estado de salud (i). Archivos de Medicina del Deporte, 20(96), 339-345. 
Michailidis, Y., Fatouros, I., Primpa, E., Avloniti, A., Chatzinikolaou, A., Barbero-Álvarez, J., ... Ambas, A. N. K. (2013). Plyometrics' trainability in preadolescent soccer athletes. Journal of Strength and Conditioning Research, 27(1), 38-49.

Monarca, H. (2012). La racionalidad de las políticas de evaluación de la calidad de la educación. Revista Iberoamericana de Educación, 59(1), 1-9.

Ogaya, S., Takahashi, H., Shioiri, M., Saito, A., \& Okajima, Y. (2012). Changes in electromyographic activity after conditioning contraction. Journal of Physical Therapy Science, 24(10), 979-983. https://doi.org/10.1589/ jpts.24.979

Organización Mundial de la Salud. (2010). Recomendaciones mundiales sobre actividad fisica para la salud. Recuperado de https://apps.who.int/iris/bitstream/handle/10665/44441/9789243599977_spa.pdf

Ozbar, N., Ates, S., \& Agopyan, A. (2014). The effect of 8-week plyometric training on leg power, jump and sprint performance in female soccer players. Journal of Strength and Conditioning Research, 28(10), 2888-2894. https://doi.org/10.1519/JSC.0000000000000541

Romano, N., Vilaça-Alves, J., Fernandes, H. M., Saavedra, F., Paz, G., Miranda, H., ... Reis, V. (2013). Effects of resistance exercise order on the number of repetitions performed to failure and perceived exertion in untrained young males. Journal of Human Kinetics, 39(1), 177-183. https://doi.org/10.2478/hukin-2013-0080

Sanchez-Medina, L., Perez, C. E., \& Gonzalez-Badillo, J. J. (2010). Importance of the propulsive phase in strength assessment. International Journal of Sports Medicine, 31(2), 123-129. https://doi.org/10.1055/s-0029-1242815

Sperlich, B., De Marées, M., Koehler, K., Linville, J., Holmberg, H.-C., \& Mester, J. (2011). Effects of 5 weeks of highintensity interval training vs. volume training in 14-year-old soccer players. Journal of Strength and Conditioning Research, 25(5), 1271-1278.

ter Stege, M. H. P., Dallinga, J. M., Benjaminse, A., \& Lemmink, K. A. P. M. (2014). Effect of Interventions on Potential, Modifiable Risk Factors for Knee Injury in Team Ball Sports: A Systematic Review. Sports Medicine, 44(10), 1403-1426. https://doi.org/10.1007/s40279-014-0216-4

Tibana, R., Prestes, J., da Cunha Nascimento, D., \& Balsamo, S. (2011). Comparison of the Number of Repetitions and Perceived Exertion Between Multi-Joint and Single-Joint Exercise at Different Intensities in Untrained Women. Brazilian Journal of Biomotricity, 5(2), 96-105.

Verkhoshansky, Y. (1999). Todo sobre el método pliométrico. Barcelona: Paidotribo

Weisstaub, G. (2015). Riesgo cardiometabólico en pediatría: obesidad infantil y condición física. Revista Chilena de Pediatria, 86(4), 221-223. https://doi.org/10.1016/j.rchipe.2015.06.010

Xu, J., Lombardi, G., Jiao, W., \& Banfi, G. (2016). Effects of Exercise on Bone Status in Female Subjects, from Young Girls to Postmenopausal Women: An Overview of Systematic Reviews and Meta-Analyses. Sports Medicine, 46(8), 1165-1182. https://doi.org/10.1007/s40279-016-0494-0

\section{BY-ND}

